\title{
WEED COMMUNITY STRUCTURE AND CHARACTERIZATION OF MACHINE-TRANSPLANTED PADDY FIELDS IN AREAS OF RICE-WHEAT ROTATION IN NORTHERN ZHEJIANG PROVINCE, CHINA
}

\author{
XU, W. D. ${ }^{1}-\mathrm{LI}, \mathrm{G} .^{2}-\mathrm{LI}, \mathrm{J}^{1}-\mathrm{LU}, \mathrm{Q}^{{ }^{*}}$ \\ ${ }^{I}$ Research Institute of Eco-Environmental, Jiaxing Academy of Agricultural Science, Jiaxing, \\ Zhejiang 314000, China \\ (e-mail:wdxu@zju.edu.cn (Xu,W.D.); liju621@sina.com (Li, J.)) \\ ${ }^{2}$ China National Rice Research Institute, Hangzhou, Zhejiang 310006, China \\ (e-mail: li@ricescience.org (Li, G.)) \\ *Corresponding author \\ e-mail: zhibao208@sina.com; phone: +86-0573-8377-8052 \\ (Received 20 $0^{\text {th }}$ Feb 2021; accepted $14^{\text {th }}$ May 2021)
}

\begin{abstract}
The study aims to characterize weed communities and identify differences in species diversity in counties using machine-transplanted paddy fields (MTPs) in areas of rice-wheat rotation in northern Zhejiang Province (NZP), P.R. China. Values of weed relative abundance (VRA), species biodiversity (SB), breadth of ecological niche (BEN) and overlap values of ecological niche (OVEN) were analyzed. The results indicated that there were 43 weed species belonging to 30 genera of 15 families, the majority being part of Gramineae and Cyperaceae. Species biodiversity in Tonglu county was significantly higher, with greater species richness, Simpson, Shannon-Wiener, and Pielou indices compared to other counties in terms of weed distribution. Hierarchical cluster analysis revealed that the weed species in NZP were divided into four principal groups. BEN analysis demonstrated that predominant weeds such as Leptochloa chinensis (L.) Nees and Echinochloa crus-galli var. mitis (Pursh) Petermann displayed higher BEN values. OVEN analysis indicated that $76.1 \%$ of the 276 pairs exhibited a niche overlap value $>0.7$, suggesting that there was great similarity in resource utilization between the majority of weed species pairs. The results will help local farmers to adopt more integrated management practices in MTPs, allowing the vegetation to provide various ecosystem services.

Keywords: weed community, biodiversity, machine-transplanted paddy fields, rice-wheat rotation areas, northern Zhejiang, P.R. China
\end{abstract}

\section{Introduction}

Rice (Oryza sativa L.) cultivation is a major agricultural activity in most region of China, with approximately 30.2 million hectares and production of $208.2 \mathrm{Mt}$ annually (NBSC, 2019). Consistent with this, rice represents the largest acreage of grain crops in Zhejiang Province, China, a crop the majority of the population consumes. The northern plain of Zhejiang Province is among the principal late japonica rice production basins in China (Yu et al., 2019). To date, with the development of China's social economy, the transfer of rural labors, and economic viability, rice production technologies are generally changing from manual labor to mechanical operation (Wang et al., 2016). Data from the Department of Agriculture and Rural Affairs of Zhejiang Province suggest that the area of machine-transplanted rice rose from $466.7 \mathrm{hm}^{2}$ to $180,000 \mathrm{hm}^{2}$ between 2005 and 2015 (Zhu et al., 2020). However, the increased proportion of mechanically transplanted rice has resulted in significant changes in the diversity of weeds, becoming a focus of study in the field of agricultural study. The dynamics of 
weeds in MTPs cropping systems have become a crucial works of agriculture protection in addition to the control of multiple classes of pest (insects, pathogens, vertebrates).

Weedy vegetation can provide vegetative diversity, usually absent in cropping systems. It has been reported that various potential benefits of weeds, such as suppression of noxious species through allelopathy, supply of shelter and food to beneficial insects, protection of the soil surface from erosion, organic matter decomposition, and sequestration of nutrients that otherwise would be leached (Gibson et al., 2006; Haddad et al., 2009; Campiglia et al., 2018; Mashavakure et al., 2019). Thus, weedy vegetation is a principal component in cropping systems that plays an irreplaceable role related to the stability of agro-ecosystems. Nevertheless, weeds differ from other plants by being more aggressive, and having particular characteristics that enable them to be more competitive. They decrease crop yield by competing for light, space, water and nutrients (Wang et al., 2007). High abundance weed species are responsible for significant losses in crop yield and quality (Thomas, 2000). Biological and cultural weed suppression are important components of integrated weed management (IWM) systems. In an IWM program, the diversity of weed management methods was found to be more important than the exclusion of any single method, such as herbicides, hand weeding, or soil tillage (Peng et al., 2019).

Chinese weed scientists have invested considerable effort in surveys of paddy weeds during recent decades. Wang et al. (2000) identified weed species and the damages they wrought in rice fields in Zhejiang in 1986-2000, the survey results showed that the weed community in late rice fields was complex and consisted of barnyardgrass (Echinochloa crusgalli), and broadleaf weeds such as alligator weed (Alternanthera philoxeroides). Currently, few previous studies have focused on weed biodiversity and community dynamics particularly in relation to MTPs in NZP environment. Varying agriculture management practices, such as cropping sequence (Ball et al., 1992), direct drilling (Muhammad et al., 2019), hand transplantation (Mohammad et al., 2006), conventional tillage (Nandan et al., 2020), and overuse of herbicides (Peng et al., 2019) have led to a great variation in the composition of weed seeds, which require study in different agroecosystems. Herbicides have represented an option for weed suppress in China since the early 1990's. Articles published on chemical control still eclipse all other weed management methods since 1995 from a global perspective (Peng et al., 2019). Many farmers rely on herbicides to reduce weed pressure in paddy fields, the primary factor controlling population dynamics in weeds (Smith et al., 2007). To ensure more accurate suppression of weeds, suitable weed survey (density, type, cover etc.) are required for the implementation of appropriate paddy management practices.

The objective of the present study was to provide baseline data to determine weed community composition and biodiversity and the dynamics of the dominant weed species for better weed management in machine-transplantation farming systems. Analysis of viable above-ground weed density can provide a reference for maintaining paddy weed biodiversity through the control of weed community assembly by human intervention. The more efficient use of resources by frequent weed species would thereby reduce the resources available to predominant weeds, increasing the reliability of such a paddy ecosystem. 


\section{Materials and methods}

\section{Study area}

Three study cities (Fig. 1) that encompass eleven counties were surveyed in NZP in China. The three areas were Jiaxing city (Xiuzhou, Jiashan, Haiyan, Pinghu, and Haining counties), Hangzhou city (Yuhang, Tonglu, and Xiaoshan counties) and Huzhou city (Changxing, Anji, and Wuxing counties) ranging from $119.52^{\circ}$ to $121.17^{\circ} \mathrm{E}$, and from $29.72^{\circ}$ to $31.07^{\circ} \mathrm{N}$. The climate in NZP is subtropical humid monsoons, with a pronounced dry/cold winter season and wet/hot summer season. Annual precipitation is $15.6-19.1 \mathrm{~mm} / 10 \mathrm{a}$, majority of which falls as rain from May to June (Sun et al., 2020). Generally, NZP has distinct seasonal characteristics, sufficient sunshine, and abundant water resources. Most farmland in NZP has been cultivated with two major crops, generally rice (Oryza sativa L.) and wheat (Triticum aestivum L.).

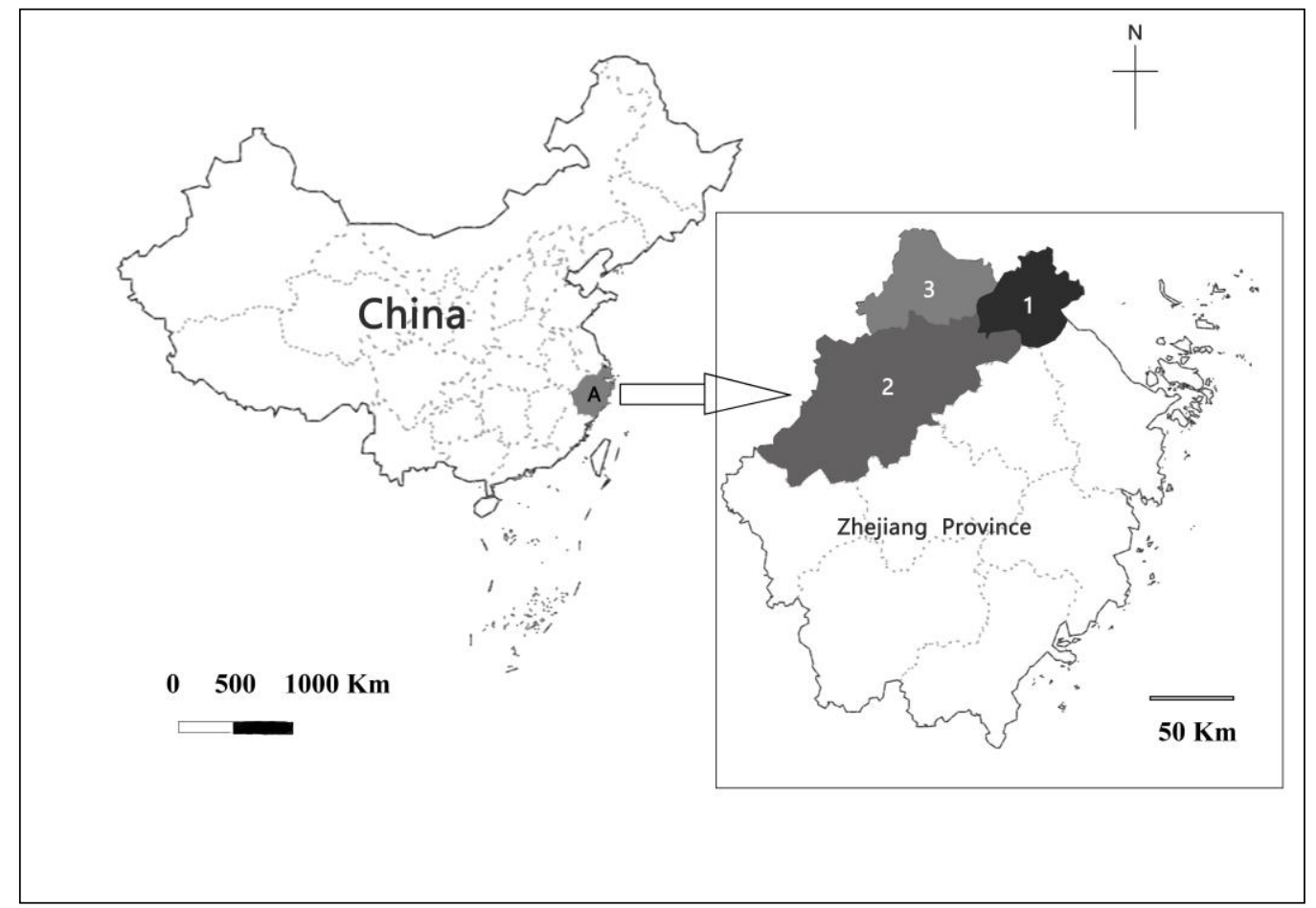

Figure 1. Map of China displaying the study region and sampling locations (1: Jiaxing, 2: Hangzhou, 3: Huzhou, A is Zhejiang Province)

\section{Investigation methodology and sites}

The weed survey was conducted during September and October, in 2018 and 2019 (during the rice's grain filling period and the weed's flowering and fruit initiation period), in MTP fields in areas of rice-wheat rotation in NZP. The study comprised 990 fields randomly distributed in the eleven counties described above (90 villages in each county), and the area of each field being $>667 \mathrm{~m}^{2}$. The GPS coordinates of the sampling sites associated with this article can be found in the Appendix 1. An inverted "W" pattern with nine sampling method was used in the survey: nine 0.5 by $0.5 \mathrm{~m}$ quadrats being set in each plot/field in accordance with Thomas (1985). The weed species, number and plant height in each quadrat were recorded. The total identified 
weeds which occurred in paddy fields were divided into four groups. The weed frequency classification of species was based on Xu et al. (2021), namely predominant weeds $(\mathrm{VRA}>5 \%)$, regional predominant weeds $(5 \% \geq \mathrm{VRA}>3 \%)$, frequent weeds $(3 \% \geq \mathrm{VRA}>1 \%)$, and infrequent weeds (VRA $\leq 1 \%)$.

\section{Statistical analysis}

The mean of two years' data in each plot were used to calculate the VRA. The VRA of each species in each county was calculated as per the formula:

$$
\begin{gathered}
\qquad R A=\frac{\mathrm{RD}+\mathrm{RH}+\mathrm{RF}}{3} \\
\text { Relative density }(\mathrm{RD})=\frac{\text { density value of species } \mathrm{i}}{\text { sum of density values for all species }} \times 100 \\
\text { Relative height }(\mathrm{RH})=\frac{\text { height value of species } \mathrm{i}}{\text { sum of height values for all species }} \times 100 \\
\text { Relative frequency }(\mathrm{RF})=\frac{\text { frequency value of species } i}{\text { sum of frequency values for all species }} \times 100
\end{gathered}
$$

The biodiversity indices of the weeds were recorded, including species richness index, Simpson's index, Shannon-Wiener diversity index, Pielou index.

Species richness index:

$$
S=\text { Number of species included in each quadrant }
$$

Simpson's index:

$$
D=1-\sum_{i=1}^{S} p_{i}^{2}
$$

where $p i=n i / N, n i$ was the percentage cover $\mathrm{m}^{-2}$ accounted for species $i$, and $N$ sum of percentage cover of all species (Molnar and Precsenyi, 2000).

Shannon-Wiener diversity index:

$$
H^{\prime}=-\sum_{i=1}^{s} P i \ln P i
$$

with $P i=N i / N$, where $N i$ is the relative frequency of species $i$ and $N$ the sum over all species (Molnar and Precsenyi, 2000).

Pielou index:

$$
J^{\prime}=\frac{H^{\prime}}{\ln S}
$$

BEN represented the Levins index: 


$$
B=\frac{1}{\sum_{j}^{2} P}
$$

where $P j$ was the proportion of species using resource $j$ (Hurlbet, 1978).

OVEN, as measured by the Pianka index:

$$
M \mathrm{jk}=\frac{\sum_{i=1}^{n} P i j P i k}{\sum_{i=1}^{n} p_{i j}^{2}}
$$

where $M j k$ represents the overlap values of ecological niche between species $k$ and $j, P i j$ or Pik was the proportions of resource $j$ or $k$ using the total resources, represented by $n$ (Pianka, 1974).

Only VRA of species that occurred more than $0.5 \%$ and the area in which it was located constituted a matrix for cluster analysis. A flexible group average method was used in clustering, with Chi-square Distance representing the distance measure. The data underwent standardization transformation.

The species biodiversity indices between counties was calculated based on of the least significant difference (LSD) test at $\mathrm{p} \leq 0.05$. All statistical analyses were performed using Microsoft Excel and SPSS statistics 20.0 (IBM Corporation, USA) for windows.

\section{Results}

\section{Weed community and relative abundance in machine-transplanted fields}

Overall, 43 weed species belonging to thirty genera of fifteen families were identified and recorded in machine-transplanted fields in rice-wheat rotation areas in NZP (Table 1). The most frequent species were from the Poaceae and Cyperaceae families, of which fifteen and eight species were represented, respectively. In addition to plants from these families, one species was from the Onagracese family, two species from the Compositae family, two species from the Lythraceae family, one species from the Pontederiaceae family, one species from the Commelinaceae family, etc., in the total recorded species. The dominant species in this group of weeds was L. chinensis $(\mathrm{L}$.) Nees, E. crus-galli var. mitis(Pursh) Petermann, and Ammannia multiflora Roxb., which were able to influence the growth and yield of rice significantly. There were eight regional predominant weeds including Ludwigia prostrate Roxb., Monochoria vaginalis (Burm. F.) Presl ex Kunth, Oryza sativa f. spontanea, Alternanthera philoxeroides (Mart.) Griseb., Leersia hexandra Swartz., Digitaria sanguinalis (L.) Scop., Spirodela polyrhiza (Linnaeus) Schleiden, Eragrostis japonica (Thunb.) Trin. These species greatly influenced the growth and development of rice in particular areas. There were 13 frequent species, including Cyperus difformis L., Murdannia triquetra (Wall. ex C. B. Clarke) Bruckn, Eclipta prostratav (L.) L., Aeschynomene indica L., Eleusine indica (L.) Gaertn., Fimbristylis littoralis Grandich, Panicum bisulcatum Thunb., Cyperus iria L., Lindernia procumbens (Krock.) Borbas, Polygonum lapathifolium L., Polygonum hydropiper L., Rotala indica (Willd.) Koehne, Echinochloa crus-galli (L.) P. Beauv. In addition, 18 infrequent weed species including Echinochloa crus-galli var. 
zelayensis(Kunth) Hithcock, Leersia japonica (Makino) Honda, Heleocharis plantaginei formis Tang et Wang., Paspalum distichum Linnaeus, Lindernia anagallis (Burm. F.) Pennell, Oenanthe javanica (B1.) DC., Echinochloa colona (Linnaeus) Link, Ammannia auriculata Willdenow, Setaria viridis (L.) Beauv., Ceratopteris thalictroides (L.) Brongn., Polygonum japonicum Meisn., Echinochloa caudata Roshev., Sagittaria pygmaea Miq., Eleocharis yokoscensis (Franchet \& Savatie) Tang \& F. T. Wang, Cyperus amuricus Maxim., Pycreus sanguinolentus (Vahl) Nees, Bidens pilosa L., Sagittaria trifolia L., Cyperus serotinus Rottb. were recorded in the present study. These weeds were unlikely to represent a major threat to the yield of rice due to their low relative abundance.

\section{Difference in weed biodiversity in different areas}

The results of the biodiversity analysis indicated that Tonglu county had a greater number of total species (thirty-four), suggesting greater weed biodiversity compared with other counties (Table 2). Haining county had poor score in the species richness index, including only twenty-one kinds of species of weeds, in addition to a relatively low Shannon index (2.9176) in all counties. Haiyan had twenty-three species of weeds but recorded the lowest Shannon index and Pielou index scores (2.8766 and 0.9174, respectively). The species richness index in other counties such as Xiuzhou, and Jiashan ranged from twenty-three to twenty-eight, with Shannon index scores ranging from were 2.9-3.2. However, no significant difference was observed in the Pielou index between any counties, which ranged from 0.93 to 0.96, except Haiyan.

\section{Weed community structure}

Hierarchical cluster analysis on the VRA (values $>0.5 \%$ ) of recorded species out of the total of forty-three species results in the identification of four groups, as displayed in Fig. 2, denoted as Xiuzhou-Haiyan-Wuxing (XHW), Jiashan-Changxing-Anji (JCA), Yuhang-Tonglu-Xiaoshan (YTX), and Pinghu-Haining (PH) Groups. Each group comprised a set of species which were similar in weed community. The weed community composition of XHW Group was Leptochloa chinensis, Echinochloa crusgalli var. mitis, Ammannia multiflora, Oryza sativa f., Alternanthera philoxeroides, Eragrostis japonica, Digitaria sanguinalis, Ludwigia prostrate, Monochoria vaginalis, Leersia hexandra, Eclipta prostrate, Aeschynomene indica, Spirodela polyrhiza, Eleusine indica. The JCA Group were dominated by Echinochloa crus-galli var. mitis, Leptochloa chinensis, Oryza sativa f., Monochoria vaginalis, Ammannia multiflora, Ludwigia prostrate, Leersia hexandra, Spirodela polyrhiza, Eragrostis japonica, Alternanthera philoxeroides, Panicum bisulcatum. The dominant species of YTX Group were Leptochloa chinensis, Echinochloa crus-galli var. mitis, Monochoria vaginalis, Ammannia multiflora, Ludwigia prostrate, Alternanthera philoxeroides, Leersia hexandra, Cyperus difformis., Murdannia triquetra. Finally, the community structure of the PH Group consisted of Leptochloa chinensis, Echinochloa crus-galli var. mitis, Ludwigia prostrate, Spirodela polyrhiza, Digitaria sanguinalis, Ammannia multiflora, Oryza sativa f., Alternanthera philoxeroides, E ragrostis japonica, Monochoria vaginalis, Leersia hexandra, Cyperus difformis, Aeschynomene indica, Murdannia triquetra. Interestingly, Echinochloa crus-galli var. mitis, Leptochloa chinensis, Ammannia multiflora, which were frequently observed in different counties, indicating that the weed communities were partly similar across the four different groups. 
Table 1. Relative abundance values (\%) of weeds in MTP fields in NZP

\begin{tabular}{|c|c|c|c|c|c|c|c|c|c|c|c|c|c|c|}
\hline $\begin{array}{c}\text { Common } \\
\text { name }\end{array}$ & $\begin{array}{c}\text { Botanical } \\
\text { name }\end{array}$ & family & Xiuzhou & Jiashan & Haiyan & Pinghu & Haining & Changxing & Anji & Wuxing & Yuhang & Tonglu & Xiaoshan & Total \\
\hline $\begin{array}{c}\text { Chinese } \\
\text { sprangletop }\end{array}$ & $\begin{array}{c}\text { Leptochloa } \\
\text { chinensis }\end{array}$ & Poaceae & 10.02 & 11.84 & 17.90 & 10.64 & 11.02 & 9.15 & 7.37 & 10.88 & 8.04 & 5.35 & 9.25 & 10.13 \\
\hline $\begin{array}{c}\text { Awnless } \\
\text { barnyardgrass }\end{array}$ & $\begin{array}{c}\text { Echinochloa } \\
\text { crus-galli var. } \\
\text { mitis }\end{array}$ & Poaceae & 10.14 & 11.20 & 8.55 & 7.26 & 7.13 & 10.36 & 8.16 & 5.56 & 6.63 & 5.05 & 7.21 & 7.93 \\
\hline $\begin{array}{c}\text { Multiflower red } \\
\text { stem }\end{array}$ & $\begin{array}{c}\text { Ammannia } \\
\text { multiflora }\end{array}$ & Lythraceae & 5.51 & 5.60 & 10.55 & 3.69 & 6.05 & 4.85 & 4.94 & 3.61 & 3.09 & 5.24 & 5.17 & 5.30 \\
\hline Waterprimrose & $\begin{array}{l}\text { Ludwigia } \\
\text { prostrate }\end{array}$ & Onagraceae & 4.74 & 6.30 & 3.99 & 8.57 & 5.21 & 4.68 & 4.38 & 3.81 & 4.38 & 4.96 & 3.46 & 4.95 \\
\hline Monochoria & $\begin{array}{c}\text { Monochoria } \\
\text { vaginalis }\end{array}$ & Pontederiaceae & 3.11 & 5.10 & 2.83 & 2.81 & 4.44 & 7.77 & 4.27 & 5.60 & 6.01 & 3.97 & 5.48 & 4.67 \\
\hline Weedy rice & Oryza sativa f. & Poaceae & 3.30 & 2.91 & 3.57 & 3.82 & 4.04 & 8.05 & 7.98 & 9.14 & 3.06 & 1.56 & 3.56 & 4.64 \\
\hline $\begin{array}{c}\text { Sessile } \\
\text { joyweed }\end{array}$ & $\begin{array}{l}\text { Alternanthera } \\
\text { philoxeroides }\end{array}$ & Amaranthaceae & 5.28 & 3.02 & 5.09 & 3.61 & 4.24 & 3.32 & 3.04 & 4.27 & 4.95 & 3.01 & 3.52 & 3.94 \\
\hline $\begin{array}{l}\text { Southern cut } \\
\text { grass }\end{array}$ & $\begin{array}{c}\text { Leersia } \\
\text { hexandra }\end{array}$ & Poaceae & 3.30 & 5.01 & 4.15 & 3.28 & 3.31 & 4.21 & 5.64 & 3.47 & 3.25 & 4.57 & 3.13 & 3.94 \\
\hline Large crabgrass & $\begin{array}{c}\text { Digitaria } \\
\text { sanguinalis }\end{array}$ & Poaceae & 4.74 & 2.82 & 4.16 & 3.81 & 5.97 & 3.89 & 3.44 & 3.97 & 3.26 & 1.99 & 2.74 & 3.71 \\
\hline Duck weed & $\begin{array}{l}\text { Spirodela } \\
\text { polyrhiza }\end{array}$ & Araceae & 3.97 & - & 5.45 & 5.75 & 5.73 & 5.73 & 6.61 & - & - & 4.69 & - & 3.45 \\
\hline Messy grass & $\begin{array}{c}\text { Eragrostis } \\
\text { japonica }\end{array}$ & Poaceae & 3.20 & 3.28 & 7.56 & 2.53 & 4.87 & 2.77 & 4.81 & 3.02 & - & 3.31 & - & 3.21 \\
\hline $\begin{array}{c}\text { Smallflower } \\
\text { umbrella sedge }\end{array}$ & $\begin{array}{l}\text { Cyperus } \\
\text { difformis }\end{array}$ & Cyperaceae & 2.11 & 3.52 & 3.26 & 3.23 & 3.09 & 2.44 & 2.02 & - & 2.78 & 2.12 & 4.74 & 2.66 \\
\hline $\begin{array}{l}\text { Triquetrous } \\
\text { murdannia }\end{array}$ & $\begin{array}{c}\text { Murdannia } \\
\text { triquetra }\end{array}$ & Commelinaceae & - & 3.15 & 1.97 & 2.84 & 3.40 & 2.31 & 2.20 & 2.15 & 2.85 & 4.43 & 2.23 & 2.50 \\
\hline Eclipta & $\begin{array}{c}\text { Eclipta } \\
\text { prostrata }\end{array}$ & Asteraceae & 4.09 & 2.72 & 2.37 & 3.89 & - & 1.88 & - & 3.20 & 2.41 & 2.97 & 3.53 & 2.46 \\
\hline $\begin{array}{l}\text { Indian } \\
\text { jointvetch }\end{array}$ & $\begin{array}{c}\text { Aeschynomene } \\
\text { indica }\end{array}$ & Fabaceae & 3.86 & - & 2.64 & 3.16 & 3.08 & 2.89 & 2.54 & 2.94 & 2.82 & 3.00 & - & 2.45 \\
\hline Goosegrass & Eleusine indica & Poaceae & 3.23 & 2.94 & 3.34 & 2.94 & - & 2.43 & 2.23 & 2.69 & 2.63 & 1.60 & 2.41 & 2.40 \\
\hline
\end{tabular}




\begin{tabular}{|c|c|c|c|c|c|c|c|c|c|c|c|c|c|c|}
\hline $\begin{array}{c}\text { Common } \\
\text { name }\end{array}$ & $\begin{array}{l}\text { Botanical } \\
\text { name }\end{array}$ & family & Xiuzhou & Jiashan & Haiyan & Pinghu & Haining & Changxing & Anji & Wuxing & Yuhang & Tonglu & Xiaoshan & Total \\
\hline $\begin{array}{c}\text { Globe } \\
\text { fringerush }\end{array}$ & $\begin{array}{c}\text { Fimbristylis } \\
\text { littoralis }\end{array}$ & Cyperaceae & 2.55 & - & 2.26 & - & 3.91 & 2.43 & 2.86 & 3.18 & 2.89 & 3.35 & 2.36 & 2.34 \\
\hline Bran millet & $\begin{array}{c}\text { Panicum } \\
\text { bisulcatum }\end{array}$ & Poaceae & 2.87 & 2.74 & - & 3.27 & - & 3.04 & 3.23 & 3.21 & 2.49 & 2.13 & 1.92 & 2.26 \\
\hline Rice flatsedge & Cyperus iria & Cyperaceae & 2.71 & 1.37 & - & - & - & 2.68 & 2.78 & 3.21 & 1.88 & 4.45 & 2.51 & 1.96 \\
\hline $\begin{array}{c}\text { Common } \\
\text { falsepimpernel }\end{array}$ & $\begin{array}{l}\text { Lindernia } \\
\text { procumbens }\end{array}$ & Linderniaceae & - & 3.18 & - & - & 1.46 & 1.47 & - & 1.44 & 2.37 & 2.54 & 2.71 & 1.38 \\
\hline Redleg & $\begin{array}{c}\text { Polygonum } \\
\text { lapathifolium }\end{array}$ & Polygonaceae & 2.58 & - & - & 3.24 & 2.49 & - & - & 3.00 & 2.29 & - & - & 1.24 \\
\hline Water pepper & $\begin{array}{l}\text { Polygonum } \\
\text { hydropiper }\end{array}$ & Polygonaceae & - & 2.30 & - & - & 2.72 & 1.67 & 1.87 & - & 2.05 & 1.98 & - & 1.15 \\
\hline Indian toothcup & Rotala indica & Lythraceae & 1.23 & 1.41 & 1.81 & 0.80 & - & - & 1.27 & 1.81 & 1.81 & 1.67 & - & 1.07 \\
\hline Barnyardgrass & $\begin{array}{c}\text { Echinochloa } \\
\text { crus-galli } \\
\text { Echinochloa }\end{array}$ & Poaceae & 1.20 & 1.20 & - & 1.50 & 1.44 & 2.30 & 2.20 & - & 1.84 & - & - & 1.06 \\
\hline Barnyardgrass & $\begin{array}{l}\text { crus-galli var. } \\
\quad \text { zelayensis }\end{array}$ & Poaceae & - & - & 1.40 & - & 1.80 & - & - & 2.50 & 2.22 & 1.20 & 1.33 & 0.95 \\
\hline False rice & $\begin{array}{c}\text { Leersia } \\
\text { japonica }\end{array}$ & Poaceae & 2.75 & - & 2.47 & - & - & 2.41 & - & 2.17 & - & - & - & 0.89 \\
\hline Wild chufa & $\begin{array}{l}\text { Heleocharis } \\
\text { plantaginei }\end{array}$ & Cyperaceae & - & 2.17 & - & - & - & 1.29 & - & 2.05 & 2.75 & 1.24 & - & 0.86 \\
\hline Knotgrass & $\begin{array}{l}\text { Paspalum } \\
\text { distichum }\end{array}$ & Poaceae & - & 2.77 & - & - & - & 2.09 & - & - & - & 1.58 & 2.30 & 0.79 \\
\hline $\begin{array}{c}\text { Long } \\
\text { falsepimpernel }\end{array}$ & $\begin{array}{c}\text { Lindernia } \\
\text { anagallis }\end{array}$ & Linderniaceae & 1.35 & - & - & 1.71 & 1.89 & - & 0.99 & - & 0.82 & 1.28 & - & 0.73 \\
\hline Water celery & $\begin{array}{l}\text { Oenanthe } \\
\text { javanica }\end{array}$ & Apiaceae & 1.38 & 1.70 & - & - & - & - & 1.40 & - & 1.46 & 1.54 & - & 0.68 \\
\hline Junglerice & $\begin{array}{l}\text { Echinochloa } \\
\text { colona }\end{array}$ & Poaceae & 2.30 & - & 2.20 & - & - & 0.96 & - & 1.80 & - & - & - & 0.66 \\
\hline Eared red stem & $\begin{array}{l}\text { Ammannia } \\
\text { auriculata }\end{array}$ & Lythraceae & - & - & - & 1.45 & - & - & - & - & 2.20 & - & 3.25 & 0.63 \\
\hline
\end{tabular}




\begin{tabular}{|c|c|c|c|c|c|c|c|c|c|c|c|c|c|c|}
\hline $\begin{array}{c}\text { Common } \\
\text { name }\end{array}$ & $\begin{array}{c}\text { Botanical } \\
\text { name }\end{array}$ & family & Xiuzhou & Jiashan & Haiyan & Pinghu & Haining & Changxing & Anji & Wuxing & Yuhang & Tonglu & Xiaoshan & Total \\
\hline Green foxtail & Setaria viridis & Poaceae & - & - & - & 2.28 & - & - & - & - & 1.98 & 1.87 & - & 0.56 \\
\hline Water fern & $\begin{array}{l}\text { Ceratopteris } \\
\text { thalictroides }\end{array}$ & Pteridaceae & - & - & 1.83 & - & - & - & 1.45 & 2.38 & - & - & - & 0.51 \\
\hline Cocoon grass & $\begin{array}{l}\text { Polygonum } \\
\text { japonicum }\end{array}$ & Polygonaceae & - & - & - & - & - & - & 2.29 & - & - & 2.29 & - & 0.42 \\
\hline $\begin{array}{c}\text { Long awn } \\
\text { barnyardgrass }\end{array}$ & $\begin{array}{c}\text { Echinochloa } \\
\text { caudate R. }\end{array}$ & Poaceae & - & - & - & 1.30 & - & - & 1.80 & - & - & 1.30 & - & 0.40 \\
\hline $\begin{array}{c}\text { Dwarf } \\
\text { arrowhead }\end{array}$ & $\begin{array}{l}\text { Sagittaria } \\
\text { pygmaea }\end{array}$ & Alismataceae & - & - & - & - & - & 0.76 & - & - & - & - & 2.00 & 0.25 \\
\hline Fescue & $\begin{array}{l}\text { Eleocharis } \\
\text { yokoscensis }\end{array}$ & Cyperaceae & - & - & - & - & - & - & - & - & - & 2.52 & - & 0.23 \\
\hline Amur sedge & $\begin{array}{l}\text { Cyperus } \\
\text { amuricus }\end{array}$ & Cyperaceae & - & - & - & - & - & - & - & - & - & - & 1.71 & 0.16 \\
\hline Red flatsedge & $\begin{array}{c}\text { Pycreus } \\
\text { sanguinolentus }\end{array}$ & Cyperaceae & - & - & 1.67 & - & - & - & - & - & - & - & - & 0.15 \\
\hline Bidens & Bidens pilosa & Asteraceae & - & - & - & - & - & - & - & - & - & 1.57 & - & 0.14 \\
\hline $\begin{array}{c}\text { Wild } \\
\text { arrowhead }\end{array}$ & $\begin{array}{l}\text { Sagittaria } \\
\text { trifolia }\end{array}$ & $\underline{\text { Alismataceae }}$ & - & - & - & - & - & - & - & - & - & - & 1.49 & 0.14 \\
\hline Water sedge & $\begin{array}{l}\text { Cyperus } \\
\text { serotinus }\end{array}$ & Cyperaceae & - & - & - & - & - & - & - & - & - & 0.99 & - & 0.09 \\
\hline
\end{tabular}


Table 2. Species diversity of weed communities in different paddy fields

\begin{tabular}{c|c|c|c|c}
\hline Areas & Species richness index & Simpson's index & Shannon index & Pielou index \\
\hline Xiuzhou & $25 \mathrm{~b}$ & $0.9557 \mathrm{a}$ & $3.0629 \mathrm{~b}$ & $0.9515 \mathrm{~b}$ \\
Jiashan & $23 \mathrm{~b}$ & $0.9458 \mathrm{a}$ & $2.9386 \mathrm{a}$ & $0.9372 \mathrm{~b}$ \\
Haiyan & $23 \mathrm{~b}$ & $0.9353 \mathrm{a}$ & $2.8766 \mathrm{a}$ & $0.9174 \mathrm{a}$ \\
Pinghu & $24 \mathrm{~b}$ & $0.9531 \mathrm{a}$ & $3.0115 \mathrm{~b}$ & $0.9476 \mathrm{~b}$ \\
Haining & $21 \mathrm{a}$ & $0.9500 \mathrm{a}$ & $2.9176 \mathrm{a}$ & $0.9583 \mathrm{~b}$ \\
Changxing & $27 \mathrm{~b}$ & $0.9553 \mathrm{a}$ & $3.0908 \mathrm{~b}$ & $0.9378 \mathrm{~b}$ \\
Anji & $26 \mathrm{~b}$ & $0.9586 \mathrm{a}$ & $3.0970 \mathrm{~b}$ & $0.9506 \mathrm{~b}$ \\
Wuxing & $25 \mathrm{~b}$ & $0.9566 \mathrm{a}$ & $3.0810 \mathrm{~b}$ & $0.9572 \mathrm{~b}$ \\
Yuhang & $28 \mathrm{~b}$ & $0.9660 \mathrm{a}$ & $3.2172 \mathrm{~b}$ & $0.9655 \mathrm{~b}$ \\
Tonglu & $33 \mathrm{c}$ & $0.9730 \mathrm{a}$ & $3.3782 \mathrm{~b}$ & $0.9662 \mathrm{~b}$ \\
Xiaoshan & $23 \mathrm{~b}$ & $0.9557 \mathrm{a}$ & $3.0075 \mathrm{~b}$ & $0.9592 \mathrm{~b}$ \\
\hline
\end{tabular}

Different lowercase letters indicate significant differences between counties counts $(p<0.05)$ for different species indices

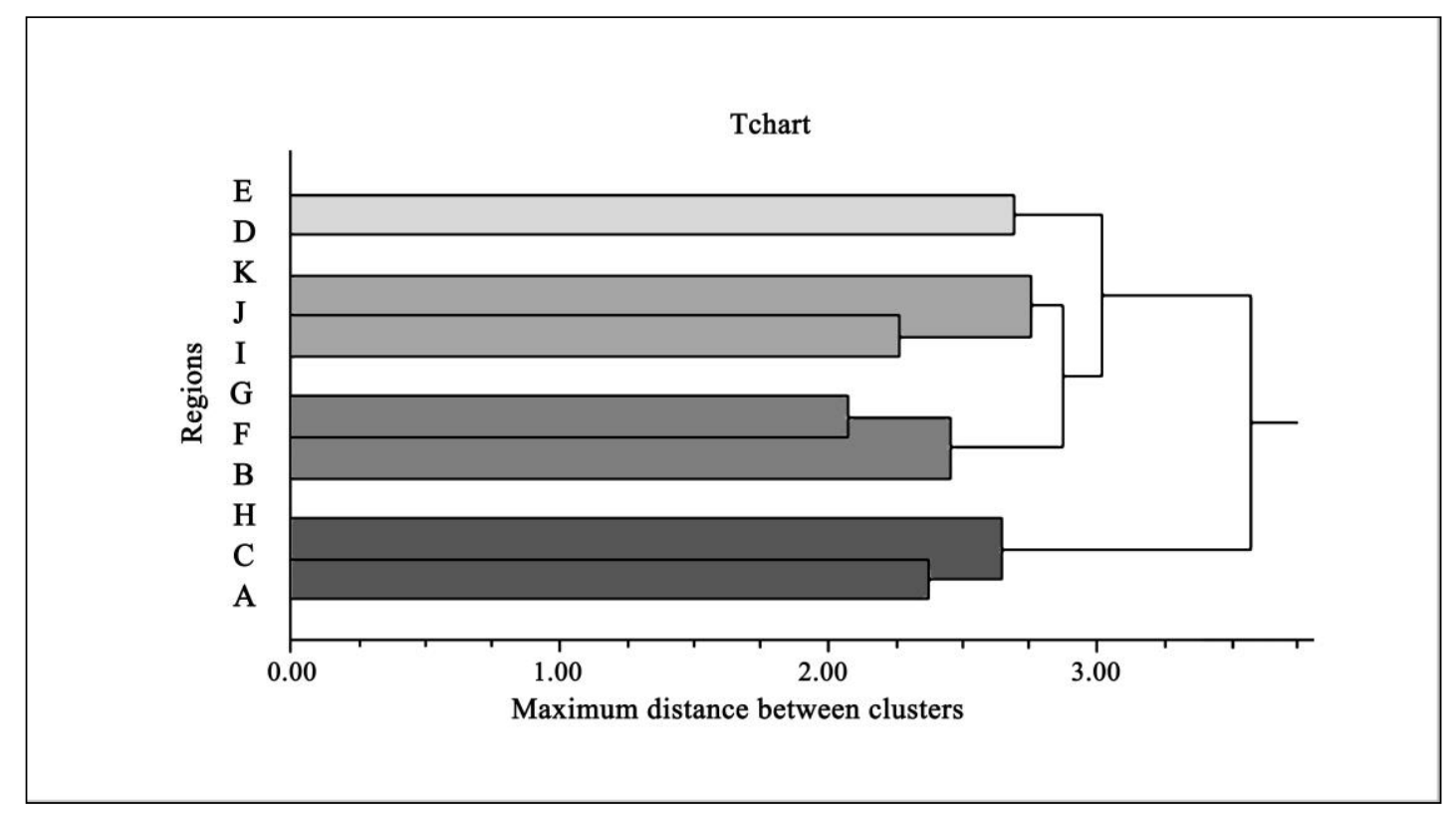

Figure 2. Dendrogram of weed communities in the different paddy regions, using cluster analysis. A is Xiuzhou; B is Jiashan; C is Haiyan; D is Pinghu; E is Haining; F Changxing; $G$ is Anji; H is Wuxing; I is Yuhang; J is Tonglu; K is Xiaoshan

\section{Niche breadth analysis for different weeds}

BEN analysis demonstrated that dominant weeds had a high breadth of ecological niche value overall, while the infrequent species had lower values (Table 3). The two perennial weeds Leersia hexandra and Alternanthera philoxeroides (Levins value were 
10.5579 and 10.5357, respectively) displayed the highest ecological breadth. Annual weeds such as Echinochloa crus-galli var. mitis, Ludwigia prostrate, Digitaria sanguinalis, Leptochloa chinensis, Monochoria vaginalis also with a broad geographic niche that had high values of breadth exceeding 10, indicating that they were adapted to a diverse array of MTP environments in different counties. In general, The Levins values of Echinochloa crus-galli var. zelayensis and Lindernia anagallis, etc. were less than 6, suggesting that the species had a limited capability to use resources in different regions in the MTP habitat.

Table 3. Breadth of ecological niche for different weeds

\begin{tabular}{|c|c|c|c|c|c|c|c|}
\hline Weeds & $\begin{array}{c}\text { Levins } \\
\text { value } \\
\end{array}$ & Weeds & $\begin{array}{c}\text { Levins } \\
\text { value }\end{array}$ & Weeds & $\begin{array}{c}\text { Levins } \\
\text { value }\end{array}$ & Weeds & $\begin{array}{c}\text { Levins } \\
\text { value } \\
\end{array}$ \\
\hline L. hexandra & 10.5579 & A. indica & 8.8722 & P. hydropiper & 5.8496 & C. thalictroides & 2.8820 \\
\hline A. philoxeroides & 10.5357 & P. bisulcatum & 8.7523 & $\begin{array}{c}\text { E. crus-galli } \\
\text { var. zelayensis }\end{array}$ & 5.5752 & A. auriculata & 2.7198 \\
\hline $\begin{array}{l}\text { E. crus-galli } \\
\text { var. mitis }\end{array}$ & 10.4114 & F. littoralis & 8.7242 & L. anagallis & 5.5679 & P. japonicum & 2.0000 \\
\hline L. prostrate & 10.2338 & $O$ sativa f. $s$. & 8.6808 & O. javanica & 4.9700 & S. pygmaea & 1.6641 \\
\hline D. san & 10.2260 & E. $p r$ & 8.5403 & P. lapathifolium & 4.9194 & E. yo & 1.0000 \\
\hline L. chinensis & 10.0930 & E. japonica & 7.8516 & H. plantaginei & 4.5869 & C. amuricus & 1.0000 \\
\hline M. vaginalis & 10.0348 & R. indica & 7.5921 & L. japonica & 3.9718 & $\begin{array}{c}P . \\
\text { sanguinolentus }\end{array}$ & 1.0000 \\
\hline A. multiflora & 9.7688 & C. iria & 7.2750 & P. distichum & 3.8526 & B. pilosa & 1.0000 \\
\hline E. indica & 9.6725 & S. polyrhiza & 6.8548 & E. colona & 3.6880 & S. trifolia & 1.0000 \\
\hline M. $t r$ & 9.3628 & $\begin{array}{l}\text { E. crus-galli } \\
\text { (L.) P. Beauv. }\end{array}$ & 6.5843 & S. viridis & 2.9786 & C serotinus & 1.0000 \\
\hline C. difformis & 9.3200 & L. procumbens & 6.4119 & E. caudate R. & 2.9245 & - & - \\
\hline
\end{tabular}

Indicator species names were abbreviated to the first letter of the Latin names. See the text above for complete names. As below

\section{Niche overlap value analysis for different weeds}

The twenty-four weed species with the highest recorded frequency had a VRA exceeding $1 \%$ and so were analyzed for niche overlap value (Table 4). The results indicated that there were $76.1 \%$ (210 pairs) of the 276 pairs with a niche overlap value exceeding 0.7 and $23.9 \%$ (66 pairs) which had a value $<0.7$, suggesting a high similarity in resource utilization between the majority of weed species pairs. For instance, the OVEN of Leptochloa chinensis with Cyperus iria, Lindernia procumbens, Polygonum lapathifolium, Polygonum hydropiper were 0.6604, 0.6686, 0.6453, and 0.6206 , for the different interspecific pairs, respectively. Furthermore, the overlap value between Polygonum lapathifolium and other majority recorded species was in each case $<0.7$, suggesting that they may compete differently with each other for resources. 
Table 4. Overlap values for ecological niche for different weeds from the different regions

\begin{tabular}{|c|c|c|c|c|c|c|c|c|c|c|c|c|c|c|c|c|c|c|c|c|c|c|c|}
\hline & $\mathrm{S} 2$ & S3 & S4 & S5 & S6 & S7 & S8 & S9 & S10 & S11 & $\mathrm{S} 12$ & S13 & S14 & S15 & S16 & S17 & S18 & S19 & S20 & S21 & S22 & $\mathrm{S} 23$ & S24 \\
\hline $\mathrm{S} 1$ & 0.9545 & 0.9735 & 0.9245 & 0.8869 & 0.847 & 0.966 & 0.9309 & 0.956 & 0.7631 & 0.899 & 0.9037 & 0.850 & 0.8527 & 0.8491 & 0.932 & 0.8163 & 0.784 & 0.6604 & 0.6686 & 0.6453 & 0.6206 & 60.8144 & $4 \longdiv { 0 . 6 8 9 5 }$ \\
\hline S2 & & 0.9389 & 0.9479 & 0.9365 & 0.8682 & 0.9516 & 0.9669 & 0.948 & 0.7823 & 0.845 & 0.9217 & 0.855 & 0.8553 & 0.8487 & 0.943 & 0.8148 & 0.880 & 0.7584 & 0.7243 & 0.5989 & 0.7198 & 80.7801 & 10.8185 \\
\hline S3 & & & 0.8881 & 0.8575 & 0.7995 & 0.9413 & 0.9374 & 0.928 & 0.8151 & 0.934 & 0.9066 & 0.851 & 0.8052 & 0.8362 & 0.894 & 0.8451 & 0.7280 & 0.6901 & 0.6585 & 0.5172 & 0.6528 & 80.7987 & 70.6512 \\
\hline $\mathrm{S} 4$ & & & & 0.8932 & 0.8241 & 0.9290 & 0.9446 & 0.932 & 0.8055 & 0.814 & 0.9070 & 0.917 & 0.8741 & 0.8772 & 0.908 & 0.7730 & 0.890 & 0.7148 & 0.7090 & 0.7126 & 0.7159 & 90.7869 & 90.8006 \\
\hline S5 & & & & & 0.9111 & 0.9180 & 0.9365 & 0.907 & 0.6840 & 0.727 & 0.8681 & 0.897 & 0.8148 & 0.8227 & 0.881 & 0.8769 & 0.889 & 0.8381 & 0.8369 & 0.5907 & 0.7692 & 20.7403 & 30.7962 \\
\hline S6 & & & & & & 0.8553 & 0.8885 & 0.884 & 0.7333 & 0.777 & 0.7109 & 0.771 & 0.7096 & 0.8334 & 0.838 & 0.8352 & 0.873 & 0.7771 & 0.5880 & 0.6163 & 0.6150 & 0.7078 & 80.7707 \\
\hline S7 & & & & & & & 0.9343 & 0.973 & 0.7595 & 0.841 & 0.8906 & 0.857 & 0.8825 & 0.9203 & 0.935 & 0.8971 & 0.839 & 0.7582 & 0.6967 & 0.7329 & 0.6592 & 20.8463 & 30.7386 \\
\hline S8 & & & & & & & & 0.923 & 0.8096 & 0.875 & 0.8920 & 0.922 & 0.8197 & 0.8612 & 0.921 & 0.8609 & 0.890 & 0.8266 & 0.7479 & 0.5532 & 0.7756 & 60.8436 & 60.7865 \\
\hline S9 & & & & & & & & & 0.8225 & 0.873 & 0.8733 & 0.849 & 0.8060 & 0.9239 & 0.874 & 0.8917 & 0.8110 & 0.6980 & 0.6448 & 0.7521 & 0.6969 & 90.7530 & 0.7904 \\
\hline S10 & & & & & & & & & & 0.872 & 0.7166 & 0.730 & 0.5683 & 0.8627 & 0.682 & 0.7382 & 0.647 & 0.5638 & 0.3304 & 0.4854 & 0.6205 & $\begin{array}{lll}5 & 0.5725\end{array}$ & 50.7486 \\
\hline S11 & & & & & & & & & & & 0.7403 & 0.767 & 0.6379 & 0.8272 & 0.778 & 0.7761 & 0.632 & 0.5785 & 0.4546 & 0.4869 & 0.6238 & 80.7650 & $0 \mid 0.6189$ \\
\hline S12 & & & & & & & & & & & & 0.869 & 0.8190 & 0.7241 & 0.859 & 0.7496 & 0.7518 & 0.6410 & 0.7668 & 0.5088 & 0.6853 & 30.6603 & 30.7146 \\
\hline S13 & & & & & & & & & & & & & 0.7613 & 0.7938 & 0.790 & 0.8251 & 0.7892 & 0.7529 & 0.8441 & 0.5553 & 0.8344 & 40.7652 & 20.6920 \\
\hline S14 & & & & & & & & & & & & & & 0.7749 & 0.931 & 0.6902 & 0.8599 & 0.7651 & 0.7003 & 0.6742 & 0.4199 & 90.7910 & $0 \mid 0.5404$ \\
\hline S15 & & & & & & & & & & & & & & & 0.824 & 0.8830 & 0.804 & 0.7369 & 0.5031 & 0.7782 & 0.6308 & 80.7923 & 30.7594 \\
\hline S16 & & & & & & & & & & & & & & & & 0.7450 & 0.9045 & 0.7676 & 0.6602 & 0.6129 & 0.5323 & 30.8686 & 60.7016 \\
\hline S17 & & & & & & & & & & & & & & & & & 0.7212 & 0.827 & 0.6803 & 0.6069 & 0.7169 & 90.7337 & 70.6514 \\
\hline S18 & & & & & & & & & & & & & & & & & & 0.8597 & 0.6806 & 0.6459 & 0.6106 & 60.7759 & 90.7915 \\
\hline S19 & & & & & & & & & & & & & & & & & & & 0.7403 & 0.4261 & 0.6124 & 40.7417 & 70.5664 \\
\hline S20 & & & & & & & & & & & & & & & & & & & & 0.3636 & 0.7576 & 60.6083 & 30.5008 \\
\hline $\mathrm{S} 21$ & & & & & & & & & & & & & & & & & & & & & 0.3592 & $2 \mid 0.5842$ & 20.5644 \\
\hline S22 & & & & & & & & & & & & & & & & & & & & & & 0.5663 & 30.7767 \\
\hline $\mathrm{S} 23$ & & & & & & & & & & & & & & & & & & & & & & & 0.5379 \\
\hline
\end{tabular}

S1-S24: L. chinensis, E. crus-galli var. mitis, A. multiflora, L. prostrate, M. vaginalis, O. sativa f., A. philoxeroides, L. hexandra, D. sanguinalis, S. polyrhiza, E. japonica, C. difformis, M. triquetra, E. prostrate, A. indica, E. indica, F. littoralis, P. bisulcatum, C. iria, L. procumbens, P. lapathifolium, P. hydropiper, R. indica, E. crus-galli (L.) P. Beauv.. respectively. Values $>0.7$ are shown in bold 


\section{Discussion}

The diversity of weed communities was due to the presence of safe sites for species establishment, and the response of weed species to agronomic disturbance (Derksen et al., 1995). Yet the weed composition had changed because of tillage systems and the use of herbicides compared with those found twenty years previously (Wang et al., 2000) in the paddy fields in northern Zhejiang Province. The monocot families Poaceae and Cyperaceae were the most species-rich lineages of the forty-three species recorded in the MTP fields in rice-wheat rotation systems in northern Zhejiang in China. The weed vegetation community structure mainly consisted of Leptochloa chinensis + Echinochloa crus-galli var. mitis + Ammannia multiflora + Ludwigia prostrate + Monochoria vaginalis $+O$. sativa f. + Alternanthera philoxeroides + Leersia hexandra + Digitaria sanguinalis + Spirodela polyrhiza + Eragrostis japonica in these areas. In comparison with a previous study a slight difference was observed in the weed assembly. Zhuang et al. found that the weed community in the paddy fields in northwest of Zhejiang Province was dominated by frequent weeds namely Echinochloa crus-galli (L.) P. Beauv. + Echinochloa crus-galli var. austrojaponensis Ohwi + Leptochloa chinensis + Ludwigia prostrate + Ammannia baccifera + Paspalum distichum + Monochoria vaginalis (Zhuang et al., 2019). In the present study, Perennial weed Paspalum distichum was not widely distributed in the mechanical transplanting habitats in northern Zhejiang. In addition, annual broadleaf Ammannia multiflora occurred at high frequency rather than Ammannia baccifera in terms of weed composition, different from observations in a previous study (Zhu et al., 2018). Leptochloa chinensis (Wassmann et al., 2009) had become problematic weeds whose population regenerated each year due to their great natality values, thereby suppressing crop yields where such weeds were allowed to mature, with excessively high numbers of seeds returning to the soil. Such high numbers result in high weed seedling emergence due to the lack of crop straw burning. In China, the government has made it illegal to burn crop residues so as to reduce greenhouse gas emissions, nutrient loss, and reduce total $\mathrm{N}$ and $\mathrm{C}$ in the topsoil (Wassmann et al., 2009). Thus annuals such as Leptochloa chinensis and Echinochloa crus-galli var. mitis predominated in the arable paddy fields. For example, in Vietnamese rice ecosystems, where the proportion of irrigated land is considerably larger than in Cambodia, Echinochloa crus-galli and Leptochloa chinensis have a negative influence on rice production (Kamoshita et al., 2014). Furthermore, there is much evidence to illustrate that the length of the life cycle of dominant weed species in a given habitat where paddy fields were plowed prior to transplantation was closely related to the frequency of soil disturbance (Sans and Ramonm, 2006). Due to this, annual grasses are powerfully competitive in paddy fields.

The emergence of early germinating weeds was inhibited by a water layer so that in some cases there was little emergence of weeds during this initial rice transplantation period in MTP field systems (Zhu et al., 2018). Because of the water conditions and the faster seedling growth in MSP fields, crop plants are however often more successful than weeds in obtaining light and space conventionally. Small biomass weeds near the soil surface, such as Lindernia anagallis and Lindernia procumbens, are sensitive to light, their growth strongly inhibited by rice vegetation-filtered light when the rice is at tillering stage because weeds are mainly adapted to open and non-shaded habitats. The population of these species decreased and ultimately became an infrequent species in the MTP habitat. Conversely, hygrophyte species Ammannia multiflora, Monochoria vaginalis, Cyperus difformis displayed high seedling emergence after severe shading or 
a layer of water. They have acclimatized to low irradiance by plastic responses that reduce the growth limiting effects of shading (Petit et al., 2018). As MSPs are widely spaced and paddy fields full of water for most of the time, there is the great possibility of weeds emerging that have adapted to wet conditions (Roy et al., 2015). Hence, they can adapt to the cultivation conditions and become the dominant weeds in transplanted paddy fields. Such weeds would significantly reduce crop yield without the appropriate integrated management in the late growth stages.

The invasion of land weeds such as Digitaria sanguinalis and Aeschynomene indica into the paddy field might otherwise have become a problem, being a considerable threat to the growth of rice (Itoh and Froud-Williams, 2014). Digitaria sanguinalis had become more abundant and problematic in MTP systems in the study. Additionally, weedy rice (Oryza sativa f.) has become among the most damaging weeds in paddy fields in Jiangsu Province, China, requiring attention to be paid to the negative impacts of plant invasions in particular ecosystems ( $\mathrm{Li}$ et al., 2014). In this paper, the weedy rice that displayed strong biological competition with cultivated rice was distributed widely in NZP, and moreover, it cannot be prevented or controlled by paddy field herbicides.

The principal purpose of the weed investigation here is to elucidate the occurrence and distribution of weed communities in the northern Zhejiang region, and to provide a reference for weed repression in MSP systems, especially for decreasing predominant weeds emergence or biomass. However, it is generally considered a trade-off between biodiversity in ecology and economic cost when controlling infrequent weeds. It is necessary to control appropriately rather than attempting to remove them completely. Kamoshita et al. (2014) proposed a hypothesis that rice ecosystems were heterogeneous and the level of production intensity relatively low, which might allow an increase in rice yield without decreasing weed diversity, to some extent. Lal et al. (2016) pointed that long fertilization practice may contribute to the development of effective weed management strategies as well as maintenance of weed diversity leading to a transition from an herbicide-dependent cropping system to one which is more environmentally friendly. Quiñones et al. (2020) had highlighted the importance of finding the balance between productive goals and biodiversity conservation in weed control management practice.

Hierarchical cluster analysis classification of the paddy vegetation weeds revealed that the eleven areas were clustered into four groups, as mentioned earlier. There were differences in the severity of some weed occurrences in these different groups. For example, the occurrence density of Oryza sativa f. in XHW and JCA Group were relatively serious in comparison with the other two groups while Cyperus difformis behave contrarily. The perennial broadleaf weed Alternanthera philoxeroides in the XHW Group had a higher frequency of occurrence than those in the JCA group. However, Booth and Swanton (2002) showed that the composition of weed communities is influenced by dramatic variations in crop characteristics, management regimes, and weed community assembly driven by the sequence of field-scale disturbance and stress that may be interpreted as assembly filters. Hence, different counties in the same city had similar weed community composition because local farmers have adopted similar agronomic measures such as the use of the same herbicide and farming techniques.

Paddies revealed higher species richness in mountain areas than those in the lowlands (Fried et al., 2018). In the present study, Tonglu county, located in a maintain area, with an upland climate and soil type, exhibited weeds species richness 33 higher 
than any other county. As a number of former findings have emphasized, soil type is the factor with the greatest influence on species distribution and weeds community structure (Pan et al., 1998; Khan et al., 2018; Mahgoub, 2019).

BEN analysis indicated that dominant weeds such as Leersia hexandra, Alternanthera philoxeroides, Echinochloa crus-galli var. mitis, Ludwigia prostrate, Digitaria sanguinalis, Leptochloa chinensis, and Monochoria vaginalis with higher frequencies have larger niches (exceeding 10), which demonstrate that they exhibited good community status within the study area. As such, the total frequency of the species in the dataset had a strong effect on the niche breadth estimates in the resource-based methods, species with higher frequencies having larger niches (Pannek et al., 2016). In contrast, when calculating the niche breadth estimates for all weed species, there were significant differences between dominant species and rare species such as Bidens pilosa, Sagittaria trifolia, Cyperus serotinus. Low niche breadth in the paddy field may simply reflect the weak utilization of resources, such as soil $\mathrm{pH}, \mathrm{C} / \mathrm{N}$ ratio, or light. Thus, rare species are vulnerable to the transformation of the paddy environment, suggesting that their complete remove is not recommended in order to protect biodiversity. For example, the niche overlap index between Leptochloa chinensis and Polygonum lapathifolium, Polygonum hydropiper were 0.6453, 0.6206, respectively, which implied that they had great difference in using resource relatively. It is worth noting that the increased density of the species of Polygonum lapathifolium or Polygonum hydropiper suppresses herbicide resistance of Leptochloa chinensis in some of the fields in China (Deng et al., 2019), with particular importance in the applied ecology of the paddy environment.

\section{Conclusions}

A paddy weed survey system methodology was shown for $2 \mathrm{yr}$ in northern Zhejiang in China, and the results of specific species provided baseline information for weed research. There were 43 weed species belonging to 30 genera of 15 families, the majority belonging to the Gramineae and Cyperaceae families in MTPs in rice-wheat rotation areas. Three predominant weed species (Leptochloa chinensis (L.) Nees, Echinochloa crus-galli (L.) var. mitis (Pursh) Petermann and Ammannia multiflora Roxb.), eight regional dominant weed species (including Ludwigia prostrate Roxb., Monochoria vaginalis (Burm. F.) Presl ex Kunth, Oryza sativa f. spontanea, Alternanthera philoxeroides (Mart.) Griseb.), and thirty-two normal weed species. The results will contribute to guiding local farmers towards more integrated management practices, and offer new insights into the association between weed control and farmland ecological protection and allowing these vegetation to provide a variety of ecosystem services. Meanwhile, it should be possible then to indicate the paddy weed community dynamics due to shifts in agricultural practices. As well as pinpointing major weed problems that require concerted research and extension activity in paddy fields.

Acknowledgments. The authors wish to acknowledge Prof. Jinwen Zhu (Ministry of Agriculture and Rural Affairs Key Lab of Molecular Biology of Crop Pathogens and Insects, Department of Plant Protection, Zhejiang University) for the weeds identification. 


\section{REFERENCES}

[1] Ball, D. A. (1992): Weeds seedbank response to tillage, herbicides, and crop rotation sequence. - Weed Science 40: 654-659.

[2] Booth, B. D., Swanton, C. J. (2002): Assembly theory applied to weed communities. Weed Science 50: 2-13.

[3] Campiglia, E., Radicetti, E., Mancinelli, R. (2018): Floristic composition and species diversity of weed community after 10 years of different cropping systems and soil tillage in a Mediterranean environment. - Weed Research 58: 273-283.

[4] Deng, W., Cai, J. X., Zhang, J. Y., Chen, Y. Y., Chen, Y. R., Di, Y. J., Yuan, S. Z. (2019): Molecular basis of resistance to accase-inhibiting herbicide cyhalofop-butyl in Chinese sprangletop (Leptochloa chinensis (L.) Nees) from China. - Pesticide Biochemistry and Physiology 158: 143-148.

[5] Derksen, D. A., Thomas, A. G., Lafond, G. P., Loeppky, H. A., Swanton, C. J. (1995): Impact of post-emergence herbicides on weed community diversity within conservationtillage systems. - Weed Research 35: 311-320.

[6] Fried, O., Kühn, L., Schrader, J., Nguyen, V. S., Bergmeier, E. (2018): Plant diversity and composition of rice field bunds in southeast Asia. - Paddy and Water Environment 16: 359-378.

[7] Gibson, R. H., Nelson, I. L., Hopkins, G. W., Hamlett, B. J., Memmott, J. (2006): Pollinator webs, plant communities and the conservation of rare plants: arable weeds as a case study. - Journal of Applied Ecology 43: 246-257.

[8] Haddad, N. M., Crutsinger, G. M., Gross, K. J., Haarstad, J., Knops, J. M. H., Tilman, D. (2009): Plant species loss decreases arthropod diversity and shifts trophic structure. Ecology Letters 12: 1029-1039.

[9] Hurlbet, S. H. (1978): The measurement of niche overlap and some relatives. - Ecology 59: 67-77.

[10] Itoh, M., Froud-Williams, R. J. (2014): Roles of emergence time and interspecific competition on the dominance and coexistence of setaria faberi and digitaria ciliaris in an orchard Weed community in Japan. - Weed Biology and Management 14: 31-42.

[11] Kamoshita, A., Araki, Y., Nguyen, Y. T. B. (2014): Weed biodiversity and rice production during the irrigation rehabilitation process in Cambodia. - Agriculture Ecosystems \& Environment 194: 1-6.

[12] Khan, K. R., Ishtiaq, M., Iqbal, Z., Alam, J., Bhatti, K. H., Shah, A. H., Farooq, M., Ali, N., Mushtaq, W., Mehmood, A., Majid, A. (2018): Effects of edaphic and physiognomic factors on species diversity, distribution and composition in reserved forest of Sathan Gali (Mansehra), Pakistan. - Applied Ecology and Environmental Research 16: 1085-1100.

[13] Lal, B., Gautam, P., Raja, R., Tripathi, R., Shahid, M., Mohanty, S., Panda, B. B., Bhattacharyya, P., Nayak, A. K. (2016): Weed seed bank diversity and community shift in a four-decade-old fertilization experiment in rice-rice system. - Ecological Engineering 86: 135-145.

[14] Li, X. Y., Qiang, S., Song, X. L., Cai, K., Sun, Y. Y., Shi, Z. H., Dai, W. M. (2014): Allele types of rc gene of weedy rice from Jiangsu province, China. - Rice Science 21: 252-261.

[15] Mahgoub, A. M. M. A. (2019): Comparative view for the impact of five eco factors on species distribution and weed community structure in Isthmus of Suez and Adjoining farmland east Nile Delta, Egypt. - Heliyon 5: e02161.

[16] Mashavakure, N., Mashingaidze, A. B., Musundire, R., Nhamo, N., Gandiwa, E., Thierfelder, C., Muposhi, V. K. (2019): Soil dwelling beetle community response to tillage, fertilizer and weeding intensity in a sub-humid environment in Zimbabwe. Applied Soil Ecology 135: 120-128. 
[17] Mohammad, S. B., Inayat, U. A., Gul, H., Abdul, A. K. (2006): Effect of establishment methods and weed management practices on some growth attributes of rice. - Rice Science 13: 131-140.

[18] Molnar, I., Precsenyi, I. (2000): Changes on the diversity (species cover) of weed communities in maize fields in eastern Hungary in 1994 and 1995. - Növénytermelés 49: 81-87.

[19] Muhammad, Z. U. H., Abdul, K., Qiang, S., Amar, M., Saddam, H., Saba, F., Zeshan, A. (2019): Weed growth, herbicide efficacy, and rice productivity in dry seeded paddy field under different wheat stubble management methods. - Journal of Integrative Agriculture 18: 907-926.

[20] Nandan, R., Singh, V., Kumar, V., Singh, S. S., Hazra, K. K., Nath, C. P., Malik, R. K., Poonia, S. P. (2020): Viable weed seed density and diversity in soil and crop productivity under conservation agriculture practices in rice-based cropping systems. - Crop Protection 136: 105210.

[21] NBSC (National Bureau of Statistics of China). (2019): National data. http://data.stats.gov.cn/easyquery.htm?cn=C01, Accessed date: 31 December 2019.

[22] Pan, D. Y., Bouchard, A., Legendre, P., Domon, D. (1998): Influence of edaphic factors on the spatial structure of inland halophytic communities: a case study in China. - Journal of Vegetation Science 9: 797-804.

[23] Pannek, A., Manthey, M., Diekmann, M. (2016): Comparing resource-based and cooccurrence-based methods for estimating species niche breadth. - Journal of Vegetation Science 27: 596-605.

[24] Peng, Q., Han, H. P., Yang, X., Bai, L. Y., Yu, Q., Powles, S. B. (2019): Quinclorac resistance in Echinochloa crus-galli from China. - Rice Science 26: 300-308.

[25] Petit, S., Cordeau, S., Chauvel, B., Bohan, D., Guillemin, J.-P., Steinberg, C. (2018): Biodiversity-based options for arable weed management. a review. - Agronomy for Sustainable Development 38: 48.

[26] Pianka, E. R. (1974): Niche overlap and diffuse competition. - Proceedings of the national academy of sciences of the United States of America 71: 2141-2145.

[27] Quiñones, A., Lattanzi, F. A., Saldain, N., Lezama, F. (2020): Weed control in natural grasslands: a case study using a perennial native forb from the south American Campos. Austral Ecology 45(7): 909-920.

[28] Roy, S., Bisht, P. S., Pandey, P. C., Paul, T. (2015): Assessment of age of seedling and weed management practices on rice yield under system of rice intensification. - PNAS, India Section B: Biological Sciences 86: 559-565.

[29] Sans, F. X., Ramonm, M. (2006): Phenological patterns in an arable land weed community related to disturbance. - Weed Research 35: 321-332.

[30] Smith, R. G., Menalled, F. D., Robertson, G. P. (2007): Temporal yield variability under conventional and alternative management systems. - Agronomy Journal 99: 1629-1634.

[31] Sun, J. J., Guo, J. H., Shen, A. H., Xu, X., Feng, H. L., Zhang, S., Yuan, W. G., Jiang, B., Wu, C. P., Wang, W. F. (2020): Composition and environmental interpretation of the communities of sassafras tzumu, a protected species, at Zhejiang province in eastern China. - Global Ecology and Conservation 24: e01218.

[32] Thomas, A. G. (1985): Weed survey system used in saskatchewan for cereal and oilseed crops. - Weed Science 33: 34-43.

[33] Thomas, V. E. (2000): Species diversity as a task for organic agriculture in Europe. Agriculture, Ecosystems \& Environment 77: 101-109.

[34] Wang, Q., He, J. H., Li, M. S., Dai, Y. Y., Shi, D., Ye, G. B. (2000): Weed species and their damage in rice fields in Zhejiang. - Acta Agricultural Zhejiangensis 12: 317-324. (In Chinese).

[35] Wang, S., Duan, L., Li, J., Tian, X., Li, Z. (2007): UV-B radiation increases paraquat tolerance of two broad leaved and two grass weeds in relation to changes in herbicide absorption and photosynthesis. - Weed Research 47: 122-128. 
[36] Wang, X. B., Yamauchi, F., Otsuka, K., Huang, J. K. (2016): Wage growth, landholding, and mechanization in Chinese agriculture. - World Development 86: 30-45.

[37] Wassmann, R., Jagadish, S. V. K., Sumfleth, K., Pathak, H., Howell, G., Ismail, A., Serraj, R., Redona, E., Singh, R. K., Heuer, S. (2009): Regional vulnerability of climate change impacts on asian rice production and scope for adaptation. - Advances in Agronomy 102: 91-133.

[38] Xu, W. D., Li, J., Lu, Q., Wang, Y. Q. (2021): Weed community composition and difference of diversity in wet direct-seeding paddy fields in rice-wheat rotation areas in northern Zhejiang province, China. - Journal of Ecology and Rural Environment 37: 5764. (In Chinese).

[39] Yu, G. X., Gong, D. K., Dai, G. J., Liu, X. P., Chen, Y., Li, H. B., Hou, S. G. (2019): Research progress of silicon fertilizer on rice. - China Rice 25: 23-25. (In Chinese).

[40] Zhu, J. W., Wang, J., DiTommaso, A., Zhang, C. X., Zheng, G. P., Liang, W., Islam, F., Yang, C., Chen, X. X., Zhou, W. J. (2018): Weed research status, challenges, and opportunities in China. - Crop Protect 134: 104449.

[41] Zhu, J. W., Liang, W., Yang, S. Y., Wang, H. F., Shi, C. H., Wang, S., Zhou, W. J., Lu Q., Islam, F., Xu, W. D., DiTommaso, A. (2020): Safety of oilseed rape straw mulch of different lengths to rice and its suppressive effects on weeds. - Agronomy 10(2): 201.

[42] Zhuang, J. W., Zhang, Z. W., Qiang, S. (2019): Weed communities in rice fields in Zhejiang province. - Journal of Plant Protection 46: 479-488. (In Chinese).

\section{APPENDIX}

This manuscript has an electronic appendix. 